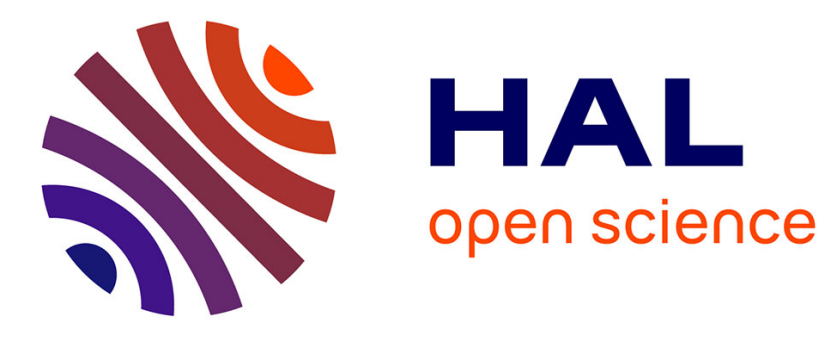

\title{
On the convergence to equilibrium for degenerate transport problems
}

Etienne Bernard, Francesco Salvarani

\section{To cite this version:}

Etienne Bernard, Francesco Salvarani. On the convergence to equilibrium for degenerate transport problems. Archive for Rational Mechanics and Analysis, 2013, 208 (3), pp.977-984. 10.1007/s00205012-0608-2 . hal-00674093v3

\section{HAL Id: hal-00674093 \\ https://hal.science/hal-00674093v3}

Submitted on 18 May 2012

HAL is a multi-disciplinary open access archive for the deposit and dissemination of scientific research documents, whether they are published or not. The documents may come from teaching and research institutions in France or abroad, or from public or private research centers.
L'archive ouverte pluridisciplinaire HAL, est destinée au dépôt et à la diffusion de documents scientifiques de niveau recherche, publiés ou non, émanant des établissements d'enseignement et de recherche français ou étrangers, des laboratoires publics ou privés. 


\title{
ON THE CONVERGENCE TO EQUILIBRIUM FOR DEGENERATE TRANSPORT PROBLEMS
}

\author{
ÉTIENNE BERNARD AND FRANCESCO SALVARANI
}

\begin{abstract}
We give a counterexample which shows that the asymptotic rate of convergence to the equilibrium state for the transport equation, with a degenerate cross section and in the periodic setting, cannot be better than $t^{-1 / 2}$ in the general case. We suggest moreover that the geometrical properties of the cross section are the key feature of the problem and impose, through the distribution of the forward exit time, the speed of convergence to the stationary state.
\end{abstract}

\section{INTRODUCTION}

The long-time behaviour of kinetic transport equations - on periodic domains or on bounded domains with specular reflection on the boundary is well known when the cross sections are bounded from below by a strictly positive constant.

In this case, the exponential decay in time of the solutions to the unique equilibrium state of the system can be obtained, with explicit rates, by the method of hypocoercivity as in $[7,12]$.

This result has, however, no obvious extension in the case of cross sections vanishing in a portion of the domain. Such a transport problem is said to be degenerate, and the characterization of the long-time asymptotics in the general case is still an open problem.

Indeed, in the regions where the cross section is zero, the problem is reduced to the free transport equation, which has no equilibrium state in a periodic setting or when the problem is defined on a bounded domain with specular reflection.

A partial answer to this question has been obtained by Desvillettes and Salvarani in [3] in a special situation, namely when the cross section vanishes at a finite number of points.

The key point of the proof in [3] is the use of the Desvillettes-Villani lemma (Theorem 6.2 in [4]), based on a pair of differential inequalities that allows them to prove a polynomial (in time) speed of convergence towards equilibrium for the solution of the transport problem.

We note that there exist other phenomena that can lead to the convergence to equilibrium of the solutions of free transport equations: we cite, for example, the interaction with the boundary of the domain in the case of diffuse reflection $[1,10]$ or the presence of a dissipating obstacle (see [9] and the references therein).

Date: April 6, 2012.

Key words and phrases. Transport equation, degenerate cross section, asymptotic behaviour. 
In this note, we consider a situation when the cross section vanishes on a set of non zero measure, and give a counterexample which shows that the $L^{2}$ distance to equilibrium cannot decay faster than $t^{-1 / 2}$ (see Theorem 3.3 below).

\section{The PROBLEM}

The problem considered here is the long-time asymptotics of the nonhomogeneous (in space) transport equation

$$
\frac{\partial f}{\partial t}+v \cdot \nabla_{x} f=\sigma(x)(\bar{f}-f)
$$

where $f:=f(t, x, v)$ represents the density of particles which at time $t \in \mathbb{R}^{+}$ and point $x \in \mathbb{T}^{d}(d \in \mathbb{N}, d \geq 2)$ move at speed 1 in the direction $v \in S^{d-1}$.

Here $S^{d-1}$ denotes the unit sphere in $\mathbb{R}^{d}$ and

$$
\bar{f}(t, x)=\frac{1}{\left|S^{d-1}\right|} \int_{S^{d-1}} f(t, x, v) d v,
$$

where $d v$ is the Euclidean surface element on $S^{d-1}$ and $\left|S^{d-1}\right|$ is the total $(d-1)$-dimensional measure of $S^{d-1}$.

The equation is set in a periodic box, that is $x \in \mathbb{T}^{d}:=\mathbb{R}^{d} / \mathbb{Z}^{d}$, and is supplemented with the initial condition

$$
f(0, x, v)=f^{\text {in }}(x, v) .
$$

We assume that $f^{\text {in }} \in L^{\infty}\left(\mathbb{T}^{d} \times S^{d-1}\right)$ and that $f^{\text {in }} \geq 0$ for a.e. $(x, v) \in$ $\mathbb{T}^{d} \times S^{d-1}$.

The nonnegative function $\sigma(x)$ designates the absorption/scattering cross section. We assume that

(1) $\sigma \in L^{\infty}\left(\mathbb{T}^{d}\right)$ and $\sigma(x) \geq 0$ for a.e. $x \in \mathbb{T}^{d}$;

(2) $\|\sigma\|_{L^{1}\left(\mathbb{T}^{d}\right)}>0$.

Since the problem (2.1)-(2.2) is a Lipschitz continuous perturbation of the free transport equation, there exists a unique mild solution $f$ of the problem (see, for example, [8]).

It is also straightforward to prove that constants are steady solutions of Equation (2.1), and that

$$
f_{\infty}=\frac{1}{\left|S^{d-1}\right|} \int_{\mathbb{T}^{d} \times S^{d-1}} f^{\text {in }}(x, v) d x d v
$$

is the unique constant solution with the same total mass (i.e. particle number) as the initial data.

Problem: Under the assumptions above, does one have

$$
\left\|f(t, \cdot, \cdot)-f_{\infty}\right\|_{L^{2}\left(\mathbb{T}^{d} \times S^{d-1}\right)}=O\left(e^{-\gamma t}\right) \quad \text { as } t \rightarrow+\infty
$$

for some $\gamma>0$ ?

We recall the following result obtained by Ukai, Point and Ghidouche [11]:

Theorem 2.1. (Ukai, Point, Ghidouche). Under the assumptions above, if $\sigma(x) \geq \sigma_{m}>0$ for a.e. $x \in \mathbb{T}^{d}$, there exist $C, \gamma>0$ such that

$$
\left\|f(t, \cdot, \cdot)-f_{\infty}\right\|_{L^{2}\left(\mathbb{T}^{d} \times S^{d-1}\right)} \leq C e^{-\gamma t}\left\|f^{\mathrm{in}}\right\|_{L^{2}\left(\mathbb{T}^{d} \times S^{d-1}\right)} .
$$


In the next section, we answer the question above in the negative and show that the Ukai-Point-Ghidouche theorem cannot be extended to the case of degenerate cross sections.

\section{A counterexample}

We consider here a case of degenerate transport where convergence to equilibrium cannot be faster than algebraic. This possibility excludes the exponential convergence under the assumptions above and without additional requirements.

Following [5], for all $r \in(0,1 / 2)$ we consider the periodic open set

$$
Z_{r}=\left\{x \in \mathbb{R}^{d}: \operatorname{dist}\left(x, \mathbb{Z}^{d}\right)>r\right\}
$$

together with the associated fundamental domain $Y_{r}=Z_{r} / \mathbb{Z}^{d}$.

A crucial tool in studying Equation (2.1) is the forward exit time for a particle starting from $x \in Z_{r}$ in the direction $v \in S^{d-1}$, defined as

$$
\tau_{r}(x, v)=\inf \left\{t>0: x+t v \in \partial Z_{r}\right\} .
$$

The forward exit time can be defined on the quotient space $Y_{r} \times S^{d-1}$ by periodicity since

$$
\tau_{r}(x+k, v)=\tau_{r}(x, v) \text { for all }(x, v) \in Z_{r} \times S^{d-1} \text { and } k \in \mathbb{Z}^{d} .
$$

On the measurable space $Y_{r} \times S^{d-1}$, equipped with its Borel $\sigma$-algebra, we define $\mu_{r}$ as the probability measure proportional to the Lebesgue measure on $Y_{r} \times S^{d-1}$, that is

$$
d \mu_{r}(y, v)=\frac{d y d v}{\left|Y_{r}\right|\left|S^{d-1}\right|}
$$

We finally define the distribution of $\tau_{r}$ under $\mu_{r}$ by

$$
\Phi_{r}(t):=\mu_{r}\left(\left\{(x, v) \in Y_{r} \times S^{d-1}: \tau_{r}(y, v)>t\right\}\right) .
$$

The distribution of forward exit time satisfies, in the periodic case, both a lower and an upper bound. This property, proved by Bourgain, Golse and Wennberg $[2,6]$ is recalled in the following theorem:

Theorem 3.1. (Bourgain, Golse, Wennberg). Let $d \geq 2$. Then there exist two positive constants $C_{1}$ and $C_{2}$ such that, for all $r \in(0,1 / 2)$ and each $t>1 / r^{d-1}$

$$
\frac{C_{1}}{r^{d-1}} t^{-1} \leq \Phi_{r}(t) \leq \frac{C_{2}}{r^{d-1}} t^{-1} .
$$

Our counterexample uses only the lower bound in Theorem 3.1. We recall that this lower bound is based on the fact that some particles never meet the scattering region, i.e. $\left\{x \in \mathbb{T}^{d}: \sigma(x)>0\right\}$, because of the presence of infinite channels [2].

Choose

$$
\sigma(x)=\mathbb{1}_{\mathbb{T}^{d} \backslash Y_{r}},
$$

and

in (2.1)-(2.2).

$$
f^{\text {in }}(x, v)=f^{\text {in }}(x)=\mathbb{1}_{Y_{r}}
$$


It is easy to prove that the only steady solution of Equation (2.1) with the same mass as the initial condition $f^{\text {in }}$ is the constant function $f_{\infty}=\left|Y_{r}\right|$. This property is a consequence of the following result:

Proposition 3.2. Let $f \in L^{2}\left(\mathbb{T}^{d} \times S^{d-1}\right)$ satisfy

$$
v \cdot \nabla_{x} f-\sigma(x)(\bar{f}-f)=0 .
$$

Then

$$
f(x, v)=\frac{1}{\left|S^{d-1}\right|} \int_{\mathbb{T}^{d} \times S^{d-1}} f(x, v) d x d v
$$

for a.e. $(x, v) \in \mathbb{T}^{d} \times S^{d-1}$.

Proof. Multiply Equation (3.1) by $f$ and integrate with respect to $(x, v) \in$ $\mathbb{T}^{d} \times S^{d-1}$. We deduce the energy estimate

$$
\int_{\mathbb{T}^{d} \times S^{d-1}} \sigma(x)(\bar{f}-f)^{2} d x d v=0 .
$$

Since $\sigma(x) \geq 0$ a.e., the previous equation implies that $\sigma(x)(\bar{f}-f)=0$ for a.e. $(x, v) \in \mathbb{T}^{d} \times S^{d-1}$.

Hence $f$ satisfies the equation $v \cdot \nabla_{x} f=0$. Therefore, by applying the Fourier transform with respect to the space variable, we obtain that $v \cdot k \hat{f}(k, v)=0$, that is $\hat{f}(k, v)=0$ for all $k$ and for a.e. $v \in S^{d-1}$.

Thus, $\operatorname{supp}(\hat{f}) \subset\{0\} \times S^{d-1}$ which means that $f=f(v)$. Again by the energy estimate, $\sigma(x) f(v)=\sigma(x) \bar{f}$ and hence, in the region $\left\{x \in \mathbb{T}^{d}\right.$ : $\sigma(x)>0\}, f(v)=\bar{f}$.

Since this region is of positive measure the announced result follows.

Since the solution of the Cauchy problem (2.1)-(2.2) satisfies

$$
\int_{\mathbb{T}^{d} \times S^{d-1}} f(t, x, v) d x d v=\int_{\mathbb{T}^{d} \times S^{d-1}} f^{\mathrm{in}}(x, v) d x d v,
$$

the only equilibrium solution to which $f$ can converge in $L^{2}\left(\mathbb{T}^{d} \times S^{d-1}\right)$ as $t \rightarrow+\infty$ is

$$
f_{\infty}=\frac{1}{\left|S^{d-1}\right|} \int_{\mathbb{T}^{d} \times S^{d-1}} f^{\text {in }}(x, v) d x d v=\left|Y_{r}\right|
$$

Thus

$$
\begin{aligned}
\int_{\mathbb{T}^{d} \times S^{d-1}}\left(f-f_{\infty}\right)^{2} d x d v & \geq \int_{Y_{r} \times S^{d-1}}\left(f-f_{\infty}\right)^{2} d x d v \\
& =\int_{Y_{r} \times S^{d-1}} \mathbb{1}_{\tau_{r}(x,-v)>t}\left(f-f_{\infty}\right)^{2} d x d v \\
& +\int_{Y_{r} \times S^{d-1}} \mathbb{1}_{\tau_{r}(x,-v) \leq t}\left(f-f_{\infty}\right)^{2} d x d v \\
& =I+J .
\end{aligned}
$$


By Duhamel's formula

$$
\begin{aligned}
f(t, x, v) & =f^{\text {in }}(x-t v, v) \exp \left(-\int_{0}^{t} \sigma(x-s v) d s\right) \\
& +\int_{0}^{t} \exp \left(-\int_{0}^{s} \sigma(x-\tau v) d \tau\right) \sigma(x-s v) \bar{f}(s, x-s v) d s \\
& \geq f^{\text {in }}(x-t v, v) \exp \left(-\int_{0}^{t} \sigma(x-s v) d s\right)
\end{aligned}
$$

since $f \geq 0$ by the maximum principle for (2.1)-(2.2).

Thus

$$
f(t, x, v) \mathbb{1}_{\tau_{r}(x,-v)>t} \geq f^{\mathrm{in}}(x-t v, v) \mathbb{1}_{\tau_{r}(x,-v)>t}
$$

since

$$
\tau_{r}(x,-v)>t \Longrightarrow \sigma(x-s v)=0 \text { for all } s \in[0, t],
$$

and therefore

$$
f(t, x, v) \mathbb{1}_{\tau_{r}(x,-v)>t} \geq \mathbb{1}_{\tau_{r}(x,-v)>t}
$$

since

$$
\tau_{r}(x,-v)>t \Longrightarrow x-t v \in Y_{r} \Longrightarrow f^{\text {in }}(x-t v, v)=1 .
$$

Hence

$$
\begin{gathered}
I=\int_{Y_{r} \times S^{d-1}} \mathbb{1}_{\tau_{r}(x,-v)>t}\left(f-f_{\infty}\right)^{2} d x d v \\
=\int_{Y_{r} \times S^{d-1}}\left(\mathbb{1}_{\tau_{r}(x,-v)>t} f-\mathbb{1}_{\tau_{r}(x,-v)>t} f_{\infty}\right)^{2} d x d v \\
\geq \int_{Y_{r} \times S^{d-1}} \mathbb{1}_{\tau_{r}(x,-v)>t}\left(1-f_{\infty}\right)^{2} d x d v \\
=\left(1-\left|Y_{r}\right|\right)^{2} \int_{Y_{r} \times S^{d-1}} \mathbb{1}_{\tau_{r}(x,-v)>t} d x d v \\
=\left(1-\left|Y_{r}\right|\right)^{2}\left|Y_{r}\right|\left|S^{d-1}\right| \Phi_{r}(t),
\end{gathered}
$$

where the inequality above follows from the fact that $f_{\infty}<1$, so that

$$
\mathbb{1}_{\tau_{r}(x,-v)>t} f_{\infty} \leq \mathbb{1}_{\tau_{r}(x,-v)>t} \leq \mathbb{1}_{\tau_{r}(x,-v)>t} f(t, x, v) .
$$

Therefore

$$
I \geq\left(1-\left|Y_{r}\right|\right)^{2}\left|Y_{r}\right|\left|S^{d-1}\right| \frac{C_{1}}{r^{d-1}} t^{-1}
$$

for all $t>r^{1-d}$.

Since

$$
J=\int_{Y_{r} \times S^{d-1}} \mathbb{1}_{\tau_{r}(x,-v) \leq t}\left(f-f_{\infty}\right)^{2} d x d v \geq 0,
$$

(3.2) implies

$$
\int_{\mathbb{T}^{d} \times S^{d-1}}\left(f-f_{\infty}\right)^{2} d x d v \geq \frac{C_{1}}{r^{d-1}}\left(1-\left|Y_{r}\right|\right)^{2}\left|Y_{r}\right|\left|S^{d-1}\right| t^{-1}
$$

or, equivalently,

$$
\left\|f-f_{\infty}\right\|_{L^{2}\left(\mathbb{T}^{d} \times S^{d-1}\right)} \geq \frac{C}{\sqrt{t}} .
$$

This particular example shows that the convergence cannot be better than polynomial in the general case. Here the $L^{2}$-norm of the difference between 
the time-dependent solution and the corresponding stationary state decays at best like $t^{-1 / 2}$.

Our result can be summarized in the following theorem:

Theorem 3.3. For all $r \in(0,1 / 2)$, there exists an initial condition $f^{\text {in }} \in$ $L^{\infty}\left(\mathbb{T}^{d} \times S^{d-1}\right)$ satisfying $f^{\text {in }}(x, v) \geq 0$ for a.e. $(x, v) \in \mathbb{T}^{d} \times S^{d-1}$ and such that, for each cross section $\sigma \in L^{\infty}\left(\mathbb{T}^{d}\right)$ satisfying $\sigma(x) \geq 0$ for a.e. $x \in \mathbb{T}^{d}$ and $\sigma(x)=0$ for a.e. $x \in Y_{r}$, the solution $f$ of the Cauchy problem (2.1)-(2.2) satisfies

$$
\left\|f-f_{\infty}\right\|_{L^{2}\left(\mathbb{T}^{d} \times S^{d-1}\right)} \geq \frac{C}{\sqrt{t}}
$$

for each $t>r^{1-d}$, where

$$
f_{\infty}=\frac{1}{\left|S^{d-1}\right|} \int_{\mathbb{T}^{d} \times S^{d-1}} f^{\text {in }}(x, v) d x d v
$$

and $C$ is a positive constant.

Remark 3.4. The initial data $f^{\text {in }}$ chosen in the proof of Theorem 3.3 is independent of the velocity variable $v$. Therefore, regularity in $v$ cannot help in obtaining exponential convergence.

Remark 3.5. The same argument shows that one can choose $f^{\text {in }} \in C^{\infty}\left(\mathbb{T}^{d}\right)$ provided that $f^{\text {in }}=0$ on $\mathbb{T}^{d} \backslash Y_{r}$. Thus regularity in $x$ cannot help either in obtaining exponential decay.

Remark 3.6. The same result holds if the isotropic scattering model considered here is replaced with a transport equation of the form

$$
\frac{\partial f}{\partial t}+v \cdot \nabla_{x} f+\sigma(x)\left[f-\frac{1}{\left|S^{d-1}\right|} \int_{S^{d-1}} p\left(v, v^{\prime}\right) f\left(t, x, v^{\prime}\right) d v^{\prime}\right]=0,
$$

where $p\left(v, v^{\prime}\right) \in L^{2}\left(S^{d-1} \times S^{d-1}\right)$ is a scattering kernel such that

$$
p\left(v, v^{\prime}\right)=p\left(v^{\prime}, v\right) \geq 0 \text { and } \frac{1}{\left|S^{d-1}\right|} \int_{S^{d-1}} p\left(v, v^{\prime}\right) d v^{\prime}=1 .
$$

Remark 3.7. The distribution of the forward exit time, induced by the geometrical properties of the scattering region, is the key ingredient in the computations.

Hence, further hypotheses on the geometry of the scattering region are necessary in order to improve the convergence rate.

Acknowledgements. The authors thank François Golse for useful discussions on the subject and Kazuo Aoki for providing us with some references.

\section{REFERENCES}

[1] Kazuo Aoki and François Golse. On the speed of approach to equilibrium for a collisionless gas. Kinet. Relat. Models, 4(1):87-107, 2011.

[2] Jean Bourgain, François Golse, and Bernt Wennberg. On the distribution of free path lengths for the periodic Lorentz gas. Comm. Math. Phys., 190(3):491-508, 1998.

[3] Laurent Desvillettes and Francesco Salvarani. Asymptotic behavior of degenerate linear transport equations. Bull. Sci. Math., 133(8):848-858, 2009.

[4] Laurent Desvillettes and Cédric Villani. On the trend to global equilibrium in spatially inhomogeneous entropy-dissipating systems: the linear Fokker-Planck equation. Comm. Pure Appl. Math., 54(1):1-42, 2001. 
[5] François Golse. On the periodic Lorentz gas and the Lorentz kinetic equation. Ann. Fac. Sci. Toulouse Math. (6), 17(4):735-749, 2008.

[6] François Golse and Bernt Wennberg. On the distribution of free path lengths for the periodic Lorentz gas. II. M2AN Math. Model. Numer. Anal., 34(6):1151-1163, 2000.

[7] Clément Mouhot and Lukas Neumann. Quantitative perturbative study of convergence to equilibrium for collisional kinetic models in the torus. Nonlinearity, 19(4):969-998, 2006.

[8] Amnon Pazy. Semigroups of linear operators and applications to partial differential equations, volume 44 of Applied Mathematical Sciences. Springer-Verlag, New York, 1983.

[9] Tetsuro Tsuji and Kazuo Aoki. On the periodic Lorentz gas and the Lorentz kinetic equation. J. Stat. Phys., 146:620-645, 2012.

[10] Tetsuro Tsuji, Kazuo Aoki, and François Golse. Relaxation of a free-molecular gas to equilibrium caused by interaction with vessel wall. J. Stat. Phys., 140(3):518-543, 2010.

[11] Seiji Ukai, Nelly Point, and Hamid Ghidouche. Sur la solution globale du problème mixte de l'équation de Boltzmann nonlinéaire. J. Math. Pures Appl. (9), 57(3):203229, 1978.

[12] Cédric Villani. Hypocoercive diffusion operators. In International Congress of Mathematicians. Vol. III, pages 473-498. Eur. Math. Soc., Zürich, 2006.

(É. B.) Ecole polytechnique, Centre de mathématiques L. Schwartz, F91128 Palaiseau cedex, \& Institut Géographique National, Laboratoire de Recherche en Géodésie, 6-8 avenue Blaise Pascal, Cité Descartes-Champs sur Marne, 77455 Marne La Valle Cedex 2

E-mail address: bernard@math.polytechnique.fr

(F. S.) Dipartimento di Matematica F. Casorati, Universitì degli Studi di Pavia, Via Ferrata 1, 27100 Pavia, Italia

E-mail address: francesco.salvarani@unipv.it 